\title{
Ostraceous and inverse psoriasis with psoriatic arthritis as the presenting features of advanced HIV infection
}

\author{
Rochelle Lorenzo $\underline{\text { Castillo }}^{1}$, MD, Geraldine Zamora Racaza ${ }^{2}$, MD, Francisca Dela Cruz Roa ${ }^{1}$, MD
}

\begin{abstract}
Knowledge of both the common and atypical presentations of human immunodeficiency virus (HIV)associated dermatoses may be helpful in arousing suspicion of HIV, especially in patients with no reported risk factors. Herein, we report the case of an otherwise healthy, nonpromiscuous 29-year-old man who presented to our institution with an eight-week history of plaques with oyster shell-like scales on the trunk, extremities and genital area. The plaques were associated with fever, and intermittent knee pain and swelling. Initial diagnostic tests were suggestive of drug hypersensitivity syndrome, and the patient's condition improved with treatment using oral prednisone. However, the lesions recurred when the dose of prednisone was tapered, even after the culprit drug had long been discontinued. Repeat skin punch biopsy and arthrocentesis revealed a diagnosis of psoriasis vulgaris with psoriatic arthritis. Due to the atypical presentation of psoriasis, the patient was counselled to undergo HIV testing, which came back positive. Clinicians should be attuned to the skin signs heralding HIV/acquired immunodeficiency syndrome, in order to facilitate early diagnosis and treatment.
\end{abstract}

Keywords: AIDS, HIV, ostraceous psoriasis, psoriasis vulgaris, psoriatic arthritis

\section{INTRODUCTION}

Dermatologic disorders occur in up to $90 \%$ of individuals with human immunodeficiency virus (HIV) infection; these disorders may be the first clinical manifestations of HIV to be recognised. ${ }^{(1,2)}$ They may also serve as useful markers of CD4+ cell decline and disease progression to acquired immune deficiency syndrome (AIDS). ${ }^{(3)}$ As such, recognition of these cutaneous clues is vital to the early diagnosis and treatment of HIV/AIDS, especially in patients with no reported risk factors.

The plethora of skin manifestations in HIV/AIDS is vast and complex, as they encompass a host of infectious, inflammatory and neoplastic disorders. ${ }^{(4)}$ Further adding to this complexity, common dermatologic conditions may have atypical presentations in individuals with HIV.(5) For example, psoriasis vulgaris, a common immune-mediated inflammatory skin disorder, classically appears as erythematous plaques with silvery-white scales on extensor surfaces of the body such as the knees and elbows (i.e. the chronic plaque type). In contrast, psoriasis vulgaris in patients with HIV may have scales that appear thick and oyster shell-like (i.e. ostraceous psoriasis) instead of silvery-white, and occur on flexural areas (i.e. inverse psoriasis) instead of on extensor surfaces. In addition, individuals with HIV often have more than one form of psoriasis, ${ }^{(6)}$ as evidenced in the present case. Thus, for patients who harbour not just lesions on their skin but also 'skeletons in the closet', the skin signs can lead clinicians to suspect HIV and recommend relevant screening.

\section{CASE REPORT}

A previously healthy 29-year-old Filipino man from Catanduanes, Philippines, presented to the Dermatology clinic of our institution for ostraceous plaques with erythematous borders on his trunk and extremities. The skin lesions had erupted eight weeks prior to his consult, initially appearing as erythematous, nontender, nonpruritic nodules over the forehead, malar area and nose; the nodules were associated with diffuse facial erythema, and scalp scaling and pustulation. He was given a seven-day course of cloxacillin for the nodules and pustules, which resolved over the next two weeks. However, the patient developed greasy yellowish scaling on his face and numerous erythematous papules, which evolved into very thickly scaled oyster shell-like plaques, over his trunk, extremities and inguinal areas. Moist, eroded papules and patches also developed on both axillae. There was associated high-grade fever and isolated, intermittent left knee pain, but no other concomitant symptoms. The patient was initiated on clindamycin by another physician, but as the treatment did not lead to a resolution of the symptoms, the patient presented at our institution for a consult. Aside from a history of adequately treated pulmonary tuberculosis, the patient's medical history was unremarkable. The patient had no chronic

\footnotetext{
${ }^{1}$ Section of Dermatology, ${ }^{2}$ Section of Rheumatology, Department of Medicine, University of the Philippines-Philippine General Hospital, Manila, Philippines Correspondence: Dr Rochelle L Castillo, Resident, Section of Dermatology, Department of Medicine, University of the Philippines-Philippine General Hospital, Taft Avenue, Manila, Philippines. shellycastillo@gmail.com
} 

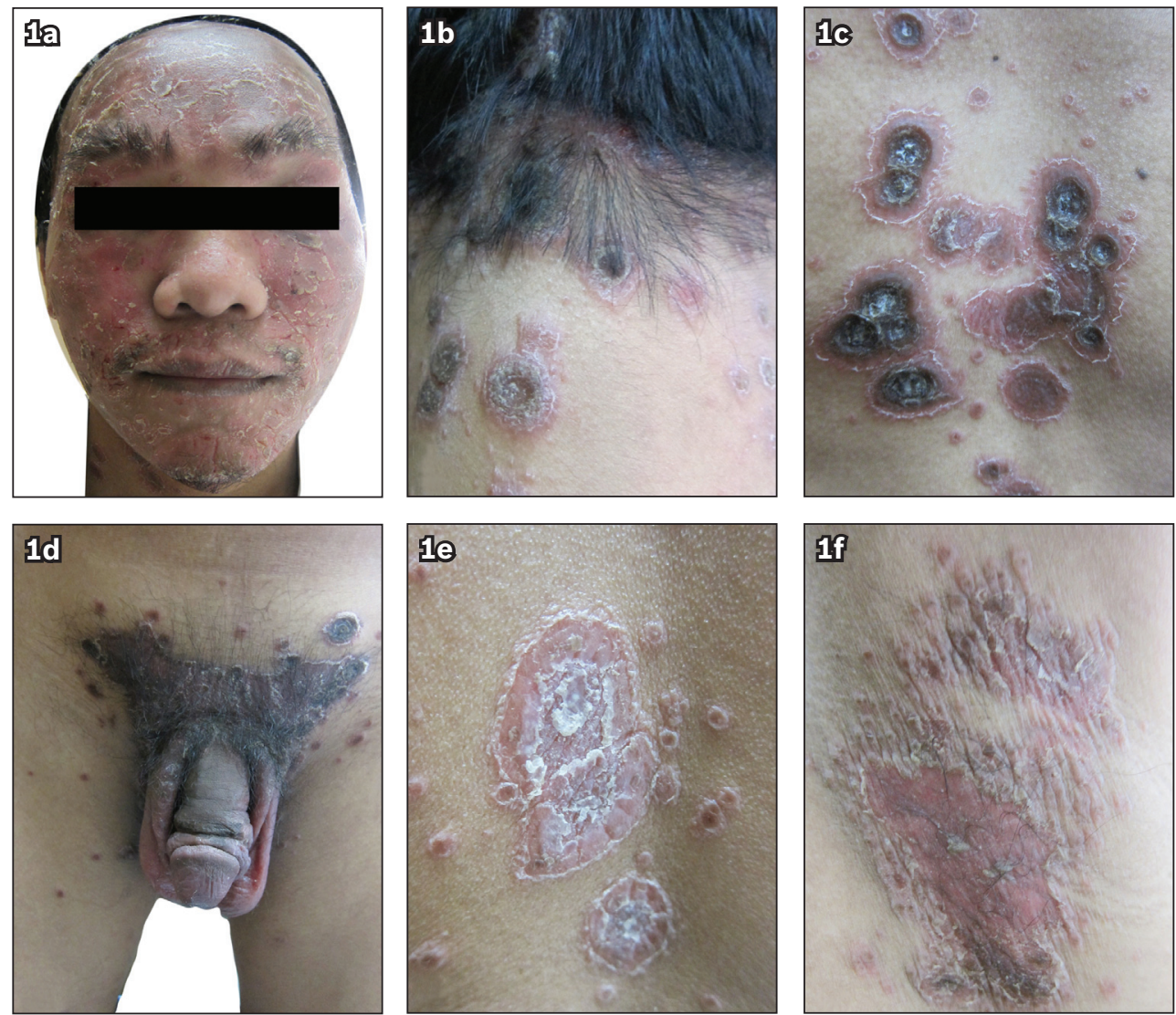

Fig. 1 Photographs show (a) diffuse areas of greasy scaling with underlying erythema and sites of fissuring on the patient's face; (b) multiple, variably-sized ostraceous plaques with underlying erythema on the nape; (c) larger ostraceous plaques with raised erythematous scaly borders interspersed with erythematous papules on the chest and abdomen; (d) confluent, crusted, erythematous to hyperpigmented plaques on the suprapubic area and diffuse erythema with fine scales on the penis and scrotum; (e) ostraceous scales on the back that had sloughed off, leaving behind erythematous annular plaques with whitish central scaling and papules; and (f) large, slightly moist, eroded patches surrounded by erythematous papules on the left axilla.

comorbidities, atopy, known food or drug allergies, or previous hospitalisations or surgeries. No other family members were similarly affected. The patient was an unemployed college undergraduate who reported to have no vices and no history of blood donation or transfusion. He denied genital, oral or anal sexual contact.

On physical examination, the patient was conversant, noncachectic (body mass index of $18.5 \mathrm{~kg} / \mathrm{m}^{2}$ ) and not in cardiorespiratory distress. His vital signs were within normal limits, and he had clear breath sounds, a regular cardiac rhythm with no murmurs, and no cervical, axillary or inguinal lymphadenopathy. Examination of his joints showed no sign of inflammation. Systemic physical examination was likewise unremarkable. Examination of the cutaneous lesions revealed diffuse areas of greasy scaling with underlying erythema and sites of fissuring on the face and scalp (Fig. 1a). There were no oral mucosal lesions. Examination of the patient's nape, chest, abdomen, back and genital region revealed multiple, variably-sized ostraceous plaques with raised erythematous scaly borders interspersed with erythematous papules (Figs. 1b-d). On several areas, the ostraceous scales had sloughed off, leaving behind erythematous annular plaques with whitish central scaling and papules (Fig. 1e). Examination of both axillae revealed large, slightly moist, eroded patches surrounded by erythematous papules (Fig. 1f). There were no other cutaneous findings. The primary working impression was that the patient had secondary syphilis or cutaneous lupus erythematosus with seborrhoeic dermatitis.

Anti-nuclear antibody test was negative and venereal disease research laboratory test was nonreactive. Initial work-up also revealed nonreactive rheumatoid factor, normocyticnormochromic anaemia, elevated aspartate transaminase (AST) and alanine transaminase (ALT) (2.5 times and 4.5 times, respectively, with an AST/ALT ratio of 0.6), and suspicious densities in the right upper lung zone on chest radiography. Skin punch biopsy of the forearm and axillae yielded a histopathologic diagnosis of subacute spongiotic dermatitis, with the following differentials: contact dermatitis, nummular dermatitis, drug reaction, or autoeczematisation dermatitis. Blood urea nitrogen, serum creatinine and urinalysis were within normal limits. Bilateral knee and elbow radiographs were largely unremarkable. Due to the constellation of clinical, histopathological and laboratory findings, the diagnosis was revised to drug hypersensitivity syndrome (based on the 

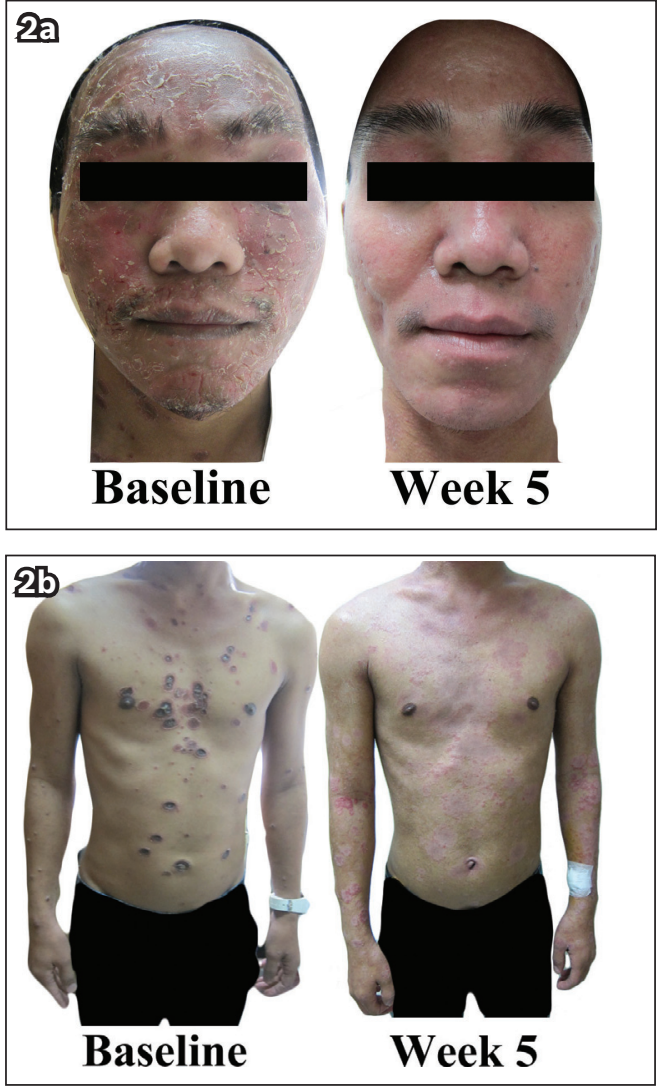

Fig. 2 Photographs show the marked improvements noted on Week 5 of treatment. (a) There was near complete resolution of the greasy scaling, erythema and fissuring on the face; and (b) thinning out of the plaques and disappearance of the ostraceous scales on the trunk.

patient's fever, rash, joint pain, transaminitis and anaemia) secondary to cloxacillin, seborrhoeic dermatitis and pulmonary tuberculosis (due to relapse or treatment failure). The patient was started on prednisone $1 \mathrm{mg} / \mathrm{kg} /$ day, ketoconazole-pyrithione zinc shampoo once daily for the scalp, hydrocortisone-fusidic acid cream twice daily for the face, and clobetasol diluted with emollients twice daily for the trunk and extremities.

After five weeks of treatment, there was a marked decrease in scalp scaling and facial erythema. Most of the ostraceous scales sloughed off, revealing thin, pinkish, annular plaques (Fig. 2). However, the lesions recurred when the dose of prednisone was tapered, even though cloxacillin had been discontinued. There was also a recurrence of knee pain; this time the pain was associated with swelling and elbow pain, prompting a referral to the Section of Rheumatology. Physical examination of the joints showed the absence of tenderness and warmth, with minimal effusions on bilateral knees. Arthrocentesis yielded $11 \mathrm{~mL}$ of synovial fluid from each knee; the fluid from the right knee was serosanguinous, light yellow and slightly opaque, while the fluid from the left knee was light yellow and slightly opaque. Both specimens showed signs of non-septic inflammation - good string sign, 1,840 × 10\% $/ \mathrm{L}$ white blood cells with neutrophilic predominance, normal synovial fluid glucose, and negative acid fast bacillus and bacterial cultures. Given the constellation of clinical and laboratory findings, a diagnosis of spondyloarthropathy, specifically

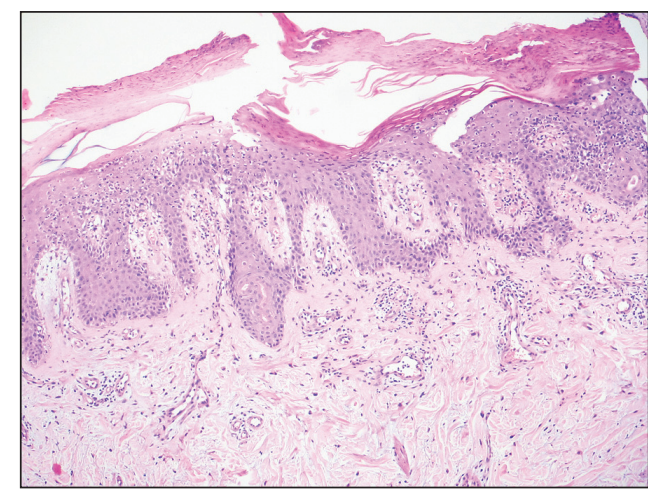

Fig. 3 skin punch biopsy shows confluent parakeratosis with neutrophils in the stratum corneum, psoriasiform epidermal hyperplasia with some squared off rete ridges, hypogranulosis, and dilated blood vessels in the dermis. Histopathological assessment was consistent with psoriasis vulgaris (Haematoxylin \& eosin, $\times 20$ ).

psoriatic arthritis, was considered. Skin punch biopsy was repeated and the findings were consistent with psoriasis (Fig. 3). The diagnosis was revised to psoriasis vulgaris with psoriatic arthritis. A resolving drug hypersensitivity syndrome could not be ruled out due to the earlier skin punch biopsy findings of a drug reaction.

Due to the patient's atypical psoriasis, severe seborrhoeic dermatitis and history of pulmonary tuberculosis, he was counselled to undergo HIV testing, which he refused. The plan of both the Sections of Dermatology and Rheumatology was to slowly taper the dose of prednisone, while initiating treatment with methotrexate at a dose of $7.5 \mathrm{mg} /$ week. The patient was subsequently lost to follow-up for a period of four weeks, during which he was unable to take all oral medications due to severe odynophagia. On follow-up, there was a recurrence of the annular psoriatic plaques (which had by then covered $60 \%$ of his body surface area), severe facial and scalp scaling, and bilateral knee pain; there was also the new finding of thick, white, curd-like plaques on the patient's hard palate, buccal mucosa and tongue. The patient finally consented to HIV testing. Two weeks later, one of the patient's family members informed our institution that the patient had expired at a private hospital from 'respiratory failure'. Results released postmortem showed that the patient was indeed HIV-1 antibody seropositive, based on enzyme-linked immunosorbent assay and Western blot.

\section{DISCUSSION}

The present case highlights the unique role skin disorders play in arousing suspicion of HIV/AIDS in otherwise asymptomatic, low-risk individuals. Early detection and diagnosis of pathologic processes that afflict the skin is highly possible, as the skin is the most accessible organ of the body. Thus, a familiarity with such pathologic findings, together with the knowledge of the strength of the associations of these findings with HIV, will allow physicians to pinpoint individuals who should undergo HIV testing, regardless of their reported risk factors (or lack thereof). 
In the present case, our patient did not appear to be at high risk for HIV infection because he denied engagement in any form of sexual intercourse and the use of intravenous drugs. The constellation of dermatologic and rheumatologic findings on physical examination was the impetus that led to the diagnosis of HIV. Other than being a means by which an early diagnosis can be reached, dermatologic diseases also serve as reliable indices of immune status, ${ }^{(3)}$ providing clinicians with a way to monitor disease progression and/or response to treatment. Certain HIV-associated cutaneous disorders have been found to correlate with CD4+ cell count; for instance, severe and refractory psoriasis and oropharyngeal candidiasis have been noted to develop at CD4+ cell counts below 500 cells $/ \mathrm{mm}^{3}$, and seborrhoeic dermatitis, at CD4+ cell counts greater than 500 cells $/ \mathrm{mm}^{3}$. ${ }^{(3)}$ Aside from the diagnostic value of dermatologic findings, the cutaneous disorders associated with HIV may lower the self-esteem of the patient and cause depression, resulting in a high risk of suicide. ${ }^{(4)}$ Thus, the dermatologic condition of individuals with HIV must be addressed and taken into consideration in the formulation of a comprehensive management plan.

The altered immune status of HIV-infected individuals may lead to unusual presentations of common dermatologic conditions, which may subsequently pose a diagnostic and therapeutic challenge. ${ }^{(5)}$ Psoriasis vulgaris in HIV patients tends to be more severe, extensive, recalcitrant and atypical, with guttate, inverse and erythrodermic subtypes occurring with the highest frequencies. ${ }^{(5-7)}$ Often, more than one form may coexist in a HIV-infected individual, ${ }^{\left({ }^{6}\right)}$ as was noted in our patient who had both inverse and ostraceous psoriasis, a rare variant of chronic plaque type psoriasis. ${ }^{(8)}$ In addition, the prevalence of psoriatic arthritis is much higher in the HIV/AIDS population than in the immunocompetent population (50\% vs $20 \%)$. $^{(9,10)}$

Treatment of psoriasis in individuals infected with HIV constitutes a distinct challenge as HIV-associated psoriasis is a T-lymphocyte-mediated disease in the setting of T-lymphocyte depletion. Many of the systemic treatments for psoriasis are immunosuppressive and can potentially lead to severe complications in HIV-infected individuals, such as progression to AIDS and development of opportunistic infections. . $^{(3,11)}$ The choice of therapeutic agents depends largely on disease severity and the patient's immune status. Topical therapy with steroids, calcipotriene, or a combination of the two, is the recommended first-line treatment for mild-to-moderate psoriasis, while phototherapy and antiretrovirals are the recommended first-line treatment for moderate-to-severe psoriasis, with oral retinoids serving as second-line treatment. ${ }^{(11)}$ In the case of a more refractory, severe cutaneous disease that is associated with arthritis, cautious use of immunosuppressives such as cyclosporine A, methotrexate, hydroxyurea and tumour necrosis factor- $\alpha$ inhibitors may be considered. ${ }^{(11)}$ Despite the availability of various treatment modalities, psoriasis in the context of HIV tends to have a chronic-recurrent character and high failure rates. ${ }^{(12)}$ However, even in the face of these difficulties, treatment should still be instituted for psoriasis and other dermatologic and rheumatologic disorders afflicting patients with HIV, as these disorders may be severely troubling to patients and adversely affect their quality of life..$^{(1,4)}$

Herein, we present the case of a 29-year-old man with no reported risk factors for HIV who developed ostraceous and inverse psoriasis with psoriatic arthritis and severe seborrhoeic dermatitis as the presenting features of HIV infection. This case highlights the importance of recognising the various dermatoses associated with HIV, especially in patients who do not report engaging in high-risk behaviours. Clinicians should be acquainted with the myriad of skin signs that can herald HIV infection, in order to facilitate early diagnosis and treatment, which will ultimately improve patient outcome.

\section{REFERENCES}

1. Fauci AS, Clifford Lane H. Human Immunodeficiency Virus Disease: AIDS and Related Disorders. In: Longo DL, Kasper DL, Larry Jameson J, Fauci AS, Hauser SL, Localzo J, eds. Harrison's Principles of Internal Medicine, 18th ed. Berkshire: McGraw Hill Medical, 2012: 557.

2. Aftergut K, Cockerell CJ. Update on the cutaneous manifestations of HIV infection. Clinical and pathologic features. Dermatol Clin 1999; 17:445-71

3. Rieger A, Minsue Chen T, Cockerell CJ. Cutaneous Manifestations of HIV Infection and HIV-related Disorders. In: Bolognia JL, Jorizzo JL, Schaffer JV, eds. Dermatology, 3rd ed. Amsterdam: Elsevier, 2012: 1285-302.

4. Cedeno-Laurent F, Gómez-Flores M, Mendez N, et al. New insights into HIV-1-primary skin disorders. J Int AIDS Soc 2011; 14:5.

5. Bartlett BL, Khambaty M, Mendoza N, et al. Dermatological management of human immunodeficiency virus. Skin Therapy Lett 2007; 12:1-3.

6. Montazeri A, Kanitakis J, Bazex J. Psoriasis and HIV infection. Int J Dermatol 1996; 35:475-9.

7. Mallon E, Bunker CB. HIV-associated psoriasis. AIDS Patient Care STDS 2000; 14:239-46.

8. Langley RG, Krueger GG, Griffiths CE. Psoriasis: epidemiology, clinical features, and quality of life. Ann Rheum Dis 2005; 64(Suppl II):ii18-23.

9. Duvic M, Johnson TM, Rapini RP, et al. Acquired immunodeficiency syndrome-associated psoriasis and Reiter's syndrome. Arch Dermatol 1987; 123:1622-32.

10. Schon MP, Boehncke WH. Psoriasis. N Engl J Med 2005; 352:1899-912.

11. Menon K, Van Voorhees AS, Bebo BF Jr, et al. Psoriasis in patients with HIV infection: from the medical board of the National Psoriasis Foundation. J Am Acad Dermatol 2010; 62:291-9.

12. Fernandes S, Pinto GM, Cardoso J. Particular clinical presentations of psoriasis in HIV patients. Int J STD AIDS 2011; 22:653-4. 